\title{
Forum
}

\section{»Deutschland zahlt mit seiner Arbeitslosigkeit den Preis für das Wirtschaftswunder« Interview mit Hajo Riese*}

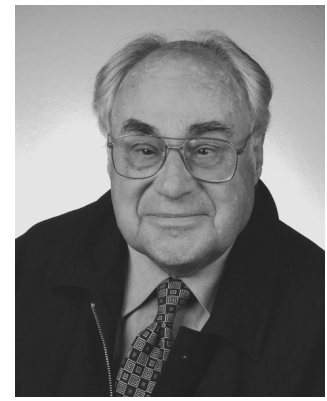

Bitte erzählen Sie uns doch zunächst ein wenig über Ihren akademischen Hintergrund, also über Ihre Ausbildung, Ihre akademischen Lehrer und Ihre beruflichen Stationen.

Ich bin Jahrgang 1933 und habe mit dem Studium 1953 in Kiel begonnen. Anschließend war ich als Assistent in Basel tätig. Dazwischen liegt ein Semester in Wien, aber dort habe ich nicht wirklich studiert - das war eher ein freies Semester, das man sich einmal zwischendurch leistet.

Kiel war seinerzeit das Zentrum der Ökonomie in Deutschland, was insbesondere an der Person von Erich Schneider lag. Schneider stand in keynesianischer Tradition, er hat in Deutschland durch seine Lehrtätigkeit und seine Buchveröffentlichungen den Anschluss an die angelsächsische und an die skandinavische Ökonomie hergestellt. Nach Basel bin ich mit Gottfried Bombach, einem Schüler von Schneider, gegangen, der sich vor allem mit Wachstumstheorien beschäftigt hat. Dort habe ich mich bei Bombach habilitiert. Danach war ich - übrigens zusammen mit Kurt Rothschild, der auch dort lehrte - drei Jahre Professor in Linz. 1970 bin ich nach Berlin gegangen, wobei ich durchaus gerne in Linz gewesen bin, aber Berlin war für mich im Alter von Mitte 30 noch einmal eine neue Herausforderung. Ich sollte vielleicht noch erwähnen, dass Anfang der I970er Jahre niemand nach Berlin gegangen ist, zum einen wegen der Studenten und zum anderen wegen der Mauer.

Wissenschaftlich bin ich der keynesianischen Tradition treu geblieben. Ich selbst sehe mich dabei nicht als Fundamentalkeynesianer, sondern bezeichne mich als Monetärkeynesianer, um mich von Paul Davidson, Hyman Minsky und anderen abzugrenzen. Ich habe in meiner Forschung mehr Wert auf die monetäre Seite als auf die einkommenstheoretische Seite gelegt, d.h. ich stelle nicht so sehr einkommens- und beschäftigungstheoretische Aspekte der Keynesschen Theorie in den Vordergrund, sondern wähle die Liquiditätstheorie des Zinses als methodischen Angelpunkt meiner Keynes-Interpretation bzw. Weiterentwicklung der Keynesschen Theorie.

Sie haben seit Anfang der I970er Jahre als aktiver Wissenschaftler miterlebt, wie sich der ökonomische Wissenschaftsbetrieb in Deutschland gewandelt hat. Während zu Beginn Ihrer Hochschullaufbahn noch der IS/LM-Keynesianismus den Hintergrund für wirtschaftspolitische

* Hajo Riese ist Professor emeritus für Wirtschaftspolitik und Wirtschaftsgeschichte an der Freien Universität Berlin. 
Debatten lieferte, dominieren heute angebotsökonomische und neoklassische Vorstellungen die Wirtschaftswissenschaften in Deutschland. Wie beurteilen Sie das und wie erklären Sie sich diesen Wandel?

Eine Rolle spielten sicher Schwächen in der Rezeption der Keynesschen Theorie, die zum Teil bei Keynes schon angelegt sind. Allerdings wäre nicht unbedingt ein Niedergang des keynesianischen Denkens seit Mitte der I970er Jahre zu erwarten gewesen. Vielmehr hätten die ökonomischen Krisen in Deutschland auch dazu führen können, dass die Genesis von Arbeitslosigkeit einen einkommens- oder beschäftigungstheoretisch fundierten Keynesianismus stimuliert. Aber das Gegenteil ist geschehen.

Im Grunde ist in der Wissenschaft eine Tendenz - ein wenig spekulativ formuliert zur Immunisierung vor der Realität feststellbar. Man darf nicht annehmen, dass die Scientific Community fasziniert ist von realen Phänomenen. Sie tut zwar so, sie meint es aber nicht wirklich. Dahinter steckt meines Erachtens auch ein nicht hinreichend geklärtes Verhältnis von Theorie und Politik. Neoklassiker argumentieren zwar markttheoretisch, aber wenn es um Wirtschaftspolitik geht, wird der Staat ins Obligo genommen; das wird insbesondere beim Pareto-Kriterium bzw. bei der Wohlfahrtstheorie deutlich. Dies gilt selbst für Walter Eucken, der dem Staat lediglich die Aufgabe zuweist, dem Markt den Ordnungsrahmen zu schaffen, jedoch zugleich dem Markt eine normative Grundlage gibt, deren Verletzung dann wiederum dem Staat eine Legitimation für Interventionen ermöglicht. Hinter diesem Widerspruch verbirgt sich ein eminentes theoretisches Problem, indem die Funktionsbedingungen des Kapitalismus und die Rolle, die die Wirtschaftspolitik in ihm zu spielen vermag, ausgeblendet werden. Die Antwort kann nur darin bestehen, den Staat als Marktteilnehmer zu fassen, der wie das einzelne Wirtschaftssubjekt ungeachtet seines größeren Einflusses den Marktbedingungen unterworfen ist. Damit kritisiere ich natürlich das in der Wirtschaftspolitik vorherrschende Ziel-Mittel-Denken, das ein Ziel(bündel) vorgibt und dann die Mittel der Zielrealisierung bereitstellt. Dabei bleibt dann undiskutiert, dass der Einsatz eines bestimmten wirtschaftspolitischen Mittels ein Marktergebnis hervorbringt, das nicht vorhersehbar war. Das gilt insbesondere für monetäre Impulse, die Preisund Mengeneffekte auslösen können, deren jeweiliges Ausmaß nicht prognostizierbar ist.

Im Mittelpunkt der wirtschaftspolitischen Debatten in Deutschland steht der Rufnach Deregulierung am Arbeitsmarkt, nach Lohnsenkungen oder zumindest nach sehr moderaten Lohnsteigerungen. Wie beurteilen Sie das? Welche Bedeutung messen Sie insbesondere dem Flächentarifvertrag bei?

Hier empfehle ich die Lektüre des zweiten Kapitels von John Maynard Keynes' General Theory. Er sagt dort sinngemäß, gegen Pigou gewendet: »Was ich jetzt sage, hättest Du, Pigou, als Neoklassiker auch schon sagen müssen, dass nämlich in Zeiten der Unterbeschäftigung die Lohnpolitik die Geldmengenpolitik ersetzt."

Keynes thematisiert an dieser Stelle, dass Lohnpolitik stets Nominallohnpolitik ist und dadurch monetären Charakter erhält. Damit wird Preisniveau stabilisierende Geldmengenpolitik der Quantitätstheorie in der keynesianischen Ökonomie dank Unterbeschäftigung durch eine produktivitätsorientierte (Nominal-)Lohnpolitik ersetzt - mit der Konsequenz, 
dass sich ein Flächentarifvertrag als ein wesentlicher Faktor einer monetären Stabilisierung erweist. Zugleich zeigt sich das Deregulierungspostulat der gängigen arbeitsmarktpolitischen Diskussion aus dem einfachen Grunde als fundamentaler theoretischer Flop, weil es nicht sauber zwischen Nominal- und Reallohn unterscheidet. Denn diese Diskussion unterstellt, dass die Tarifparteien Reallöhne aushandeln, somit ein Konnex zwischen Nominal- und Reallohn besteht. Aber das Preisniveau als Bindeglied zwischen Nominal- und Realsphäre ist kein politischer Parameter, sondern ein Marktergebnis. Damit ist jedoch der Reallohn (anders als der Nominallohn) ebenfalls ein Marktergebnis, folglich von den Tarifparteien überhaupt nicht aushandelbar.

In Zeiten der Vollbeschäftigung hat die Quantitätstheorie selbstverständlich für die Bestimmung des Preisniveaus ihre Berechtigung, einfach deshalb, weil es eine Interdependenz zwischen Geldmenge und Preisniveau gibt. In Zeiten der Unterbeschäftigung sieht die Sache aber ganz anders aus, was eben von Keynes im zweiten Kapitel der General Theory herausgearbeitet wird. In Zeiten der Unterbeschäftigung ist der Flächentarifvertrag Stabilisator eines monetären Prozesses, dessen Preis- und Mengeneffekte als Marktergebnis indeterminiert bleiben.

Trotz einer leichten Belebung steckt Deutschland im Grunde im sechsten Jahr einer wirtschaftlichen Stagnation. Was sind aus Ihrer Sicht die tiefer liegenden Ursachen?

Nach meiner Auffassung ist die Ursache der Arbeitslosigkeit die sinkende Wachstumsrate des Sozialproduktes, die durchaus einhergeht mit einer sinkenden Wachstumsrate der Arbeitsproduktivität, wobei aber letztere Rate über der ersteren liegt. Die Wachstumsrate des Sozialproduktes würde ich natürlich keynesianisch, das heißt einkommenstheoretisch, erklären. Auch bei sinkender Wachstumsrate des Sozialproduktes sind die Unternehmen gezwungen, weiter zu rationalisieren. Formal marshallianisch kann man meine Überlegung dadurch ausdrücken, dass der Profit durch eine Quasirente ersetzt wird: Der Profit sinkt, und dies wird durch eine Quasirente überkompensiert.

Für Deutschland gilt speziell, dass es mit seiner Arbeitslosigkeit den Preis für das Wirtschaftswunder zahlt. Die so genannten Wirtschaftswunderjahre - das heißt die Wachstumsphase der Nachkriegszeit - waren durch das Wachstum des sekundären Sektors, also des Industriesektors, gekennzeichnet. Dies stößt seit längerer Zeit offenbar an Grenzen und erklärt die sinkenden Wachstumsraten in der Bundesrepublik. Aber der sekundäre Sektor hat eben ein besonderes Potential des technischen Fortschritts und damit für die Steigerung der Arbeitsproduktivität. Wir haben mithin in Deutschland eine besondere Konstellation, die zum Verlust von Arbeitsplätzen führt. Länder wie die USA weisen eine ganz andere Struktur auf, und zwar insbesondere durch einen größeren Dienstleistungssektor.

Deutschland zeichnet sich durch eine starke Exportorientierung aus, gleichzeitig besteht ein Mangel an Binnennachfrage. Die traditionelle keynesianische Position würde nun darin bestehen, die Binnennachfrage zu stimulieren. In Hinblick auf solch eine Politik bin ich aber sehr skeptisch - vor allem in Hinblick auf höhere Lohnabschlüsse. Natürlich sollten Lohnsteigerungen die Höhe des Produktivitätsfortschritts widerspiegeln, aber ein Anstieg des Lohns darüber hinaus führt zu Inflation und hat mithin keine Kaufkraft stei- 
gernde Wirkung. Gegen staatliche Ausgabenpolitik habe ich in gewissem Rahmen natürlich nichts einzuwenden, aber auch sie hat Grenzen.

$\mathrm{Zu}$ Wirtschaftswachstum und Arbeitslosigkeit allgemein möchte ich noch eine banale, aber doch wichtige Bemerkung ergänzen: Seit den I980er und I990er Jahren beobachten wir weltweit sinkende Wachstumsraten. Im Grunde sind Zeiten der Vollbeschäftigung, wirtschaftshistorisch betrachtet, besondere zeitliche Abschnitte, während Arbeitslosigkeit eigentlich der Normalzustand ist. Das entspricht im Übrigen dem liquiditätspräferenztheoretischen Ansatz der keynesianischen Ökonomie, den Keynes selbst durch seine Vollbeschäftigungspolitik vulgarisiert hat.

Warum gelingt es in Deutschland bisher nicht, über ein stärkeres Wachstum des Dienstleistungssektors das Wirtschaftswachstum zu erhöhen und damit Anschluss an die Wachstumsraten in vergleichbaren Industrieländern zu erreichen?

Ehrlich gesagt, ich weiß es nicht. Mir kommt es im Übrigen in erster Linie darauf an, gegen den Mainstream der unseligen wirtschaftspolitischen Diskussion, der die mangelnde Flexibilität des Arbeitsmarktes für die unbefriedigende Situation der Wirtschaft in der Bundesrepublik Deutschland verantwortlich macht, das simple wachstumstheoretische Argument einer Diskrepanz zwischen Produktivitätsfortschritt und Produktionswachstum zu setzen. Dieses Argument ist für eine reife Volkswirtschaft, der es nicht gelingt, die Dominanz des Industriesektors zurückzudrängen, typisch. Warum dies derzeit in Deutschland nicht gelingt, ist vielleicht eher ein sozialpsychologisches als ein ökonomisches Phänomen. Auf jeden Fall sind die ökonomischen Konsequenzen beträchtlich. Das ist alles, was ich dazu zu sagen habe.

Die Europäische Zentralbank bestimmt seit 1999 die Geschicke der europäischen Geldpolitik. Dazu drei Fragen: Sehen Sie Unterschiede in Hinblick auf den geldpolitischen Spielraum zwischen der Deutschen Bundesbank und der Europäischen Zentralbank? Wie beurteilen Sie die Stellung des Euro in der Währungshierarchie? Und wie bewerten Sie die recht überraschende Berufung von Jürgen Stark zum Chefvolkswirt der EZB?

Zunächst zu Ihrer letzten Frage. Es ist durchaus interessant, wie hier Personalpolitik betrieben worden ist. Zuerst hieß es, dass Stark nicht die Position von Otmar Issing als Chefvolkswirt einnehmen werde. Sehr verständlich, denn Stark ist nun wirklich nicht in der Lage, die überragende Rolle von Issing zu übernehmen. Bildlich gesprochen kann Stark den Mantel von Issing nicht ausfüllen. Doch dann wollte man Stark wohl nicht diskreditieren. Deshalb hat man den Arbeitsbereich von Issing aufgeteilt und Stark einen Teil von Issing übertragen.

$\mathrm{Zu}$ Ihren ersten beiden Fragen: Ich sehe einen entscheidenden Unterschied zwischen der EZB und der Bundesbank, der zwar banal, aber bisher unzureichend verstanden ist. Die EZB hat einen wesentlich weiteren Spielraum, als ihn die Bundesbank je hatte. Der Spielraum der EZB ähnelt dem des FED. Dies liegt natürlich daran, dass der Euro eine andere Stellung im Währungswettbewerb aufweist, als es seinerzeit für die Deutsche Mark galt. Der Euro verfügt sicher nicht ganz über die Bedeutung des US-Dollar, aber er ist doch eine Schlüsselwährung. 
Die Rolle der DM wird demgegenüber häufig falsch eingeschätzt. Die DM ist immer eine Nischenwährung geblieben. Die Bundesbank wollte auch nie, dass sie zur Schlüsselwährung wird. Ihre Stärke beruhte darauf, dass sie eine Nischenwährung mit hoher Qualität war. Diese Qualität konnte sie nur durch strikte monetäre Disziplin aufrechterhalten, sprich: durch Preisniveaustabilität. Das bedeutet, dass die Preisniveaustabilität polit-ökonomisch gesehen werden muss und nicht diese furchtbaren Argumente in den Vordergrund geschoben werden dürfen, die Deutschen seien durch die Inflation der I920er Jahre geschädigt und hätten ein anderes Preisbewusstsein als Menschen in anderen Ländern.

Wie sieht es aus mit einem gemeinsamen Währungsraum für schwächere Länder? Konkret: Halten Sie eine Übernahme des Euro durch weitere osteuropäische Länder, etwa Polen und Ungarn, für sinnvoll und wie schätzen Sie die Risiken ein?

Die Risiken sind enorm hoch. Die Integration der Ex-DDR in die deutsche Wirtschaft ist hier von exemplarischer Bedeutung. Man hat seinerzeit nicht vorhergesehen, dass die westlichen Firmen, die genügend freie Kapazitäten hatten, die Ostmärkte erobern und die autochthonen Strukturen zerstören würden.

Die entscheidende Frage ist, ob die osteuropäischen Länder aufgrund der Lohndifferenzen auf Dauer nur als verlängerte Werkbänke fungieren werden - das spiegelt sich in den gegenwärtigen Verlagerungsdebatten wider -, oder ob sie die Chance erhalten, das reale Lohngefälle auf Dauer zu nivellieren. Ich bin in dieser Frage, wie gesagt, eher skeptisch.

Eine entscheidende Rolle bei der Integration der osteuropäischen Staaten wird letztlich die Wechselkursfrage spielen, d.h. die osteuropäischen Länder müssten durch eine Unterbewertung ihrer Währung in die Lage versetzt werden, Überschüsse in ihrer Leistungsbilanz zu erzielen. Im Umkehrschluss bedeutet dies natürlich, dass sich Deutschland vom exportorientierten Wachstum verabschieden und seine Binnennachfrage stimulieren müsste. Solche Überlegungen werden in Deutschland natürlich so aufgenommen, als wollte man eine heilige Kuh schlachten. Man sollte sich aber nicht der Illusion hingeben, dass eine Stimulierung der Inlandsnachfrage die Lösung des deutschen Beschäftigungsproblem ist: Letztlich würde die Binnen- die Außennachfrage substituieren. Eine Einkommens- oder Wachstumslücke lässt sich so nicht schließen.

Aus weltwirtschaftlicher Perspektive muss man im Übrigen die USA loben, da deren Leistungsbilanzdefizit einen unheimlich starken Stabilisator für das Weltwährungs- und das Weltwirtschaftssystem insgesamt darstellt. Das entlastet auch Länder wie Deutschland. Der deutsche Außenhandelsüberschuss ist wohlfahrtsökonomisch im Übrigen vollkommen absurd, da es sinnlos ist, den Wohlstand anderer Nationen zu produzieren. Vielmehr kommt es darauf an, die Marktbedingungen zu schaffen, die es erlauben, eigenen Wohlstand zu produzieren. Das beschränkt die Marktlogik eines Außenhandelsüberschusses auf eine typische Entwicklungsphase, wie sie derzeit Länder der Dritten Welt und Osteuropas aufweisen, in der es darauf ankommt, weltmarktfähige Güter herzustellen.

Sie können für sich immerhin in Anspruch nehmen, als einer der ganz wenigen deutschsprachigen Ökonomen so etwas wie eine eigene Schule etabliert zu haben. 
Das mit der Schule hat natürlich einen ganz banalen Grund: Ich habe wie jeder Professor Schüler. Das ist ein Funktionsprinzip des akademischen Betriebs. Der Unterschied besteht aus meiner Sicht darin, dass ich dem ansonsten ganz normalen Verhältnis einen Namen gegeben habe. Meinen akademischen Einfluss halte ich aber für eher begrenzt.

In Ihren eigenen theoretischen Arbeiten haben Sie immer wieder die Notwendigkeit gleichgewichtstheoretischer Überlegungen betont. Hierin unterscheiden Sie sich fundamental von anderen keynesianischen Strömungen, z.B. dem keynesianischen Fundamentalismus. Können Sie dies erläutern?

Meines Erachtens ist es sinnvoll, aus methodischen Gründen von vollkommener Konkurrenz auszugehen. Den Grund für ein solches Vorgehen hat Don Patinkin am Anfang seiner Money, Interest, and Prices genannt: Es geht darum, die unabhängigen von den abhängigen Variablen zu trennen. Dazu braucht man einen theoretischen Kern und nicht den Rückzug der Forschung auf eine unvollkommene Welt und die Existenz von Rigiditäten. Deshalb halte ich auch Karl Marx für einen liberalen Ökonomen, weil er den theoretischen Kern in der klassischen Produktionspreistheorie geortet hat - und sogar die Defizite dieser Theorie erkannte, auch wenn er die damit einhergehenden Probleme nicht zu lösen vermochte.

Verzichtet man jedoch auf gleichgewichtstheoretische Überlegungen, dann entsteht eine Makroökonomie auf der Basis von Verhaltenshypothesen und des Verzichts auf eine entscheidungslogische bzw. präferenztheoretische Fundierung. Verhaltenstheoretische Aussagen enthalten aber immer ein Moment der Beliebigkeit.

Damit im Zusammenhang steht die Frage nach dem Mikrofundament der keynesianischen Theorie. Notwendig ist so etwas wie die Bestimmung von Güter- und Vermögenspreisen, allen voran der Gleichgewichts- bzw. Durchschnittsprofitrate. Sie wird im Keynesianismus durch die Liquiditätstheorie des Zinses erreicht, das heißt, die Gleichgewichtsprofitrate wird durch den Zins festgelegt. Um ein Gleichgewichtspreissystem zu bestimmen, lässt sich meiner Ansicht nach auf Piero Sraffa zurückgreifen. Der bestehende Freiheitsgrad der Verteilung kann so geschlossen werden, dass der Zins die gleichgewichtige Profitrate bestimmt. Der Reallohn wird so zum Residuum. Und damit bestätigt sich zugleich die Logik der keynesianischen Ökonomie, dass Arbeitslosigkeit nichts mit dem Arbeitsmarkt zu tun hat.

Das Interview führten Kai Eicker-Wolf und Sabine Reiner im Juni 2006.

\section{Ausgewählte Publikationen von Hajo Riese}

Theorie der Produktion und Einkommensverteilung, in: Kyklos 198I • Geldökonomie, Keynes und die Anderen, Kritik der monetären Grundlagen der Orthodoxie, in: Ökonomie und Gesellschaft, Jahrbuch I, Die Neoklassik und ihre Herausforderungen, Frankfurt a.M. / New York I983 - Keynes, Schumpeter und die Krise, in: Konjunkturpolitik 1986 - Theorie der Inflation, Tübingen 1986 - Wider den Dezisionismus der Theorie der Wirtschaftspolitik, in: Winfried Vogt (Hg.), Politische Ökonomie heute, Regensburg 1988 • Stabilitätspolitik in einem dollar-euro-zentrierten Weltwährungssystem, in: Wilhelm Nölling/Karl Albrecht Schachtschneider/Joachim 
Starbatty (Hg.), Währungsunion und Weltwirtschaft, Stuttgart I999 • Money, Capital and System Transformation, London / Basingstoke: MacMillan 200I • Grundlegungen eines monetären Keynesianismus, Ausgewählte Schriften 1964-I999, 2 Bde., Marburg 200 I

\section{Zur Bedeutung der Arbeitsmarktbedingungen für die Erklärung der Arbeitslosigkeit}

\section{Labour Market Conditions and Unemployment}

Jürgen Kromphardt*, Stephanie Schneider*

In der öffentlichen Diskussion - und leider auch in wirtschaftswissenschaftlichen Debatten wird häufig behauptet, dass Arbeitslosigkeit nur durch das Geschehen auf dem Arbeitsmarkt erklärt werden könne. Deshalb müsse der Arbeitsmarkt reformiert werden, um die Unterbeschäftigung zu verringern. Diese Fixierung auf den Arbeitsmarkt als Ursache der Arbeitslosigkeit hat Solow als Ergebnis eines Fehlschlusses kritisiert. Dies sei so, als ob jemand bei einem platten Reifen das Loch nur an der Stelle vermute, an der der Reifen platt gedrückt auf der Fahrbahn aufliege, obwohl sich das Loch, das zum Entweichen der Luft geführt habe, durchaus an einer anderen Stelle befinden könne (Solow 2000: 4).

Woher rührt nun diese häufig anzutreffende Fixierung auf den Arbeitsmarkt und die Arbeitsmarktbedingungen? Offensichtlich wird das Symptom, die Unterbeschäftigung auf dem Arbeitsmarkt, für die Ursache gehalten, die eigentlich erst noch zu ergründen wäre. Konsequenterweise werden dann Änderungen bei den Arbeitsmarktbedingungen gefordert. Zwei von diesen stehen im Vordergrund: a) die strukturellen Diskrepanzen zwischen Arbeitsangebot und Arbeitsnachfrage und b) die Höhe des Reallohns.

\section{Zur strukturellen Arbeitslosigkeit}

Von struktureller Arbeitslosigkeit spricht man dann, wenn Angebot und Nachfrage in ihrer Struktur nicht zusammen passen, so dass es gleichzeitig Arbeit suchende Erwerbspersonen und Arbeitskräfte suchende Unternehmen gibt. Die Strukturdiskrepanzen können sich vor allem auf das Alter, die Qualifikation, die Berufsgruppe und die Region beziehen. Am gravierendsten sind die Differenzen in der Qualifikation. In Deutschland übersteigt das Angebot an gering qualifizierten Arbeitskräften (statistisch abgrenzbar z.B. durch das Fehlen eines berufsbildenden Abschlusses) die Nachfrage nach solchen Arbeitskräften deutlich. Dabei ist die zu geringe Arbeitsnachfrage in diesem Bereich u.a. auf die Qualifikations-

* Technische Universität Berlin. 